\title{
Cárie dentária e fatores associados entre adolescentes no norte do estado de Minas Gerais, Brasil: uma análise hierarquizada
}

\author{
Tooth decay and associated factors among adolescents in the north \\ of the State of Minas Gerais, Brazil: a hierarchical analysis
}

\author{
Marise Fagundes Silveira ${ }^{1}$ \\ Rafael Silveira Freire ${ }^{2}$ \\ Marcela Oliveira Nepomuceno ${ }^{2}$ \\ Andréa Maria Eleutério de Barros Lima Martins ${ }^{1}$ \\ Luiz Francisco Marcopito ${ }^{3}$
}

${ }^{1}$ Programa de PósGraduação em Ciências da Saúde, Universidade Estadual de Montes Claros. Av. Dr. Rui Braga s/n, Vila Mauricéia. 39401-089 Montes Claros MG Brasil. ciaestatistica@yahoo.com.br ${ }^{2}$ Faculdades Integradas Pitágoras de Montes Claros. ${ }^{3}$ Departamento de Medicina Preventiva, Universidade Federal de São Paulo.

\begin{abstract}
This is a cross-sectional population-based study ( $n=763)$ conducted in the north of the State of Minas Gerais, which aimed to investigate the prevalence of tooth decay among adolescents and to identify the potential determinants of same. Probability sampling by conglomerates in multiple stages was used. Trained and calibrated professionals performed the data collection by means of intraoral examination and interviews in the previously selected households. In the analysis of the determinant factor for the presence of tooth decay, hierarchical binary logistic regression models were used. The prevalence of tooth decay, decayed, missing and filled teeth were $71.3 \%, 36.5 \%, 55.6 \%$ and $16 \%$, respectively. The following averages were observed: DMFT (3.4 teeth), number of decayed ( 0.8 teeth), restored (2.4 teeth) and missing (0.2 teeth). The incidence of tooth decay was higher among adolescents who stated they were black/indigenous/brown $(\mathrm{OR}=$ 1.76), lived in crowded households $(O R=2.4)$, did not regularly visit or had never been to a dentist $(O R=1.9)$, used public or philanthropic services $(O R=1,8)$, had smoking habits $(O R=4.1)$, consumed alcohol $(O R=1.8)$, perceived their oral health negatively $(O R=5.9$ and $O R=1.9)$ and had toothac in the last six months $(O R=2.0)$.
\end{abstract}

Key words Oral health, Tooth decay, Social determinants of health, Adolescents
Resumo Estudo transversal de base populacional $(n=763)$, realizado na região norte do estado de Minas Gerais, que objetivou investigar a prevalência de cárie dentária entre adolescentes e identificar seus potenciais determinantes. Adotouse amostragem probabilística por conglomerado em múltiplos estágios. Profissionais treinados e calibrados realizaram os exames intrabucais e entrevistas nos domicílios. Foi utilizado modelo de regressão logística para identificar os fatores associados à cárie dentária. As prevalências de cárie dentária, dente cariado, restaurado e perdido foram $71,3 \%, 36,5 \%, 55,6 \%$ e $16, \%$, respectivamente. Observaram-se as seguintes médias: indice CPOD (3,4 dentes), número de dentes cariados ( 0,8 dente), restaurados (2,4 dentes) e perdidos ( 0,2 dente). As chances de cárie foram maiores em adolescentes que se autodeclararam negro/indio/ pardo $(O R=1,6)$, moravam em domicílios com maior aglomeração $(O R=2,4)$, não fizeram visitas regulares ao dentista ou nunca foram ao dentista $(O R=1,9)$, utilizaram os serviços públicos ou filantrópicos $(O R=1,8)$, possuíam hábitos tabagistas $(O R=4,1)$, consumiam bebida alcoólica $(O R=1,8)$, que autoperceberam negativamente sua saúde bucal $(O R=5,9 e O R=1,9)$ e sentiram dor nos dentes nos últimos seis meses $(O R=2,0)$. Palavras-chave Saúde bucal, Cárie dentária, Determinantes sociais da saúde, Adolescentes 


\section{Introdução}

A cárie dentária representa o problema de saúde bucal mais importante e prevalente no Brasil, sendo considerado um problema de saúde públi$\mathrm{ca}^{1}$. Na adolescência, a prevalência de cárie atinge o dobro dos valores registrados para a idade-índice de 12 anos $^{2}$, e entre adultos e idosos produz perdas dentárias em magnitude expressiva, levando milhões de brasileiros à mutilação e ao edentulismo parcial ou total ${ }^{2}$.

Ressalta-se, entretanto que, no Brasil, levantamentos epidemiológicos nacionais entre adolescentes de 15 a 19 anos revelaram uma diminuição na prevalência e experiência de cárie dentária. Os resultados de 1986 apontaram um índice CPOD médio de $12,7^{3}$,já em 2002/2003 foi constatado um CPO-D médio de 6,2, com $88,9 \%$ dos adolescentes com experiência de cárie dentária ${ }^{4}$. No último inquérito, em 2010, foi identificado CPO-D médio de 4,3 com 76,1\% dos examinados com experiência de cárie ${ }^{5}$. Vale ressaltar que os critérios de exame não foram os mesmos nesses três levantamentos. No levantamento de $1986^{3}$ o diagnóstico de cárie foi mais abrangente, houve utilização da sonda exploradora como auxiliar de diagnóstico. Em $2003^{4}$ e $2010^{5}$, os critérios foram menos "sensíveis", considerando cárie somente lesões cavitadas e lesões secundárias. Sendo assim, parte do declínio do CPOD pode ser atribuída a essas mudanças nos critérios de diagnóstico da cárie ${ }^{6,7}$. Outros fatores apontados como responsáveis pelo declínio da cárie foram a fluoretação das águas de abastecimento público, dentifrícios com flúor ${ }^{2}$, mudanças no consumo de açúcar, e melhorias nas condições socioeconômicas ${ }^{6,8}$.

A cárie dentária é a doença bucal mais estudada em todo o mundo, entretanto há uma escassez de estudos populacionais com esse tema entre adolescentes de 15 a 19 no Brasil, já que a maioria das pesquisas avaliou crianças com 12 anos de idade ${ }^{9,10}$. Nessa fase, por se tratar de uma doença cumulativa, a cárie dentária pode apresentar distribuição média e prevalências inferiores às registradas entre adultos e idosos ${ }^{5}$. Entretanto, a adolescência é tida como um período de maior comportamento de risco para cárie dentária, em decorrência do precário controle de placa e da redução dos cuidados com a higiene bucal, agravados pela maior independência em relação ao consumo de uma alimentação mais açucarada ${ }^{11}$. Por outro lado, a adolescência é também a fase na qual o jovem pode adquirir um aprendizado relacionado a atitudes e comportamentos positi- vos que persistirão no futuro, representando um momento fundamental para a promoção da saú$\mathrm{de}^{12}$. Os efeitos negativos da cárie dentária sobre a vida dos adolescentes incluem dificuldade de mastigar, diminuição do apetite, perda de peso, dificuldade para dormir, irritabilidade, baixa autoestima, e diminuição do rendimento escolar ${ }^{13}$. Portanto, ressalta-se a relevância de estudos epidemiológicos que investiguem a saúde bucal desse grupo etário.

A cárie dentária apresenta uma etiologia multifatorial e já foi descrita como uma doença social. Sua prevalência tem como determinantes fatores biológicos, alimentares, comportamentais e socioeconômicos, bem como fatores de acesso a bens de consumo e a serviços de saúde ${ }^{6}$. Não foram encontrados estudos que tenham avaliado de forma concomitante a existência de associação entre a cárie dentária e os fatores anteriormente elencados, considerando ainda fatores subjetivos. Sugere-se que a cárie pode estar associada à autopercepção da saúde bucal, da mastigação, da fala e da aparência dos dentes e gengivas, assim como ao relato de experiência dolorosa nos dentes e gengivas nos últimos seis meses.

Neste contexto, considerando a escassez de dados populacionais sobre a prevalência de cárie dentária entre adolescentes na região norte mineira, o objetivo do presente estudo foi descrever as condições de saúde bucal relacionada à cárie dentária de adolescentes dessa região, bem como identificar os seus potenciais determinantes.

\section{Metodologia}

Estudo transversal de base populacional, cuja população-alvo foi constituída pelos adolescentes de 15 a 19 anos, residentes nas áreas urbana e rural de um município de porte médio, no norte do estado de Minas Gerais (MG), nos anos de $2008 / 2009$. Essa faixa etária foi preconizada pela OMS como adequada para representar a população de adolescentes em estudos epidemiológicos de saúde bucal ${ }^{14}$.

\section{Plano amostral}

O tamanho da amostra foi calculado visando estimar parâmetros populacionais com prevalência de 0,50 , com intervalo de $95 \%$ de confiança e nível de precisão de 5,0\%. Foi adotado um fator de correção para o efeito de desenho (deff) igual a 2, para proteger o impacto do delineamento por conglomerados sobre a precisão fixada ini- 
cialmente, admitindo o processo de amostragem como aleatória simples. Os cálculos evidenciaram a necessidade de se examinar e entrevistar 637 indivíduos. Sob a hipótese de $20 \%$ de não cobertura (recusa, domicílio vago, não localização do indivíduo sorteado após três visitas), ficou estabelecido um tamanho amostral de 761 observações.

$\mathrm{Na}$ área urbana, as unidades amostrais foram selecionadas, utilizando-se a amostragem probabilística por conglomerado em dois estágios. No primeiro estágio, foram selecionados por amostragem aleatória simples 52 dos 276 setores censitários urbanos. No segundo, por amostragem aleatória simples, foi selecionada uma fração amostral das quadras em cada um dos 52 setores sorteados (proporcional ao número de quadras do setor), obtendo-se em média sete quadras/ setor. Todos os domicílios das quadras selecionadas foram sequencialmente visitados e seus residentes pertencentes à faixa etária estudada foram convidados a participar da pesquisa.

$\mathrm{Na}$ zona rural, optou-se por uma amostra probabilística por conglomerados em um único estágio, em que as unidades primárias de amostragem foram constituídas pelas áreas rurais. Foram sorteadas duas das onze áreas rurais identificadas, e todos os domicílios situados a uma distância de até 500 metros de uma instituição de referência (escola) foram selecionados ${ }^{15}$. Os indivíduos com idade entre 15 e 19 anos foram convidados a participar do inquérito.

\section{Sistema de ponderação}

Para incorporar a estrutura do plano amostral complexo na análise dos dados, cada entrevistado foi associado a um peso $w$, que correspondeu ao inverso de sua probabilidade de inclusão na amostra $(f)^{16}$. Na área urbana, a probabilidade de inclusão foi obtida pelo produto da probabilidade de inclusão em cada um dos dois estágios ( $f=$ $f_{1} \times f_{2}$ ). Sendo $f_{1}=$ probabilidade de inclusão no primeiro estágio ( $\mathrm{n}^{\circ}$ de setores sorteados $/ \mathrm{n}^{\circ}$ total de setores) e $f_{2}=$ probabilidade de inclusão no segundo estágio ( $\mathrm{n}^{\circ}$ de quadras sorteados/ $\mathrm{n}^{\circ}$ total de quadras do setor). Além disso, considerou-se também a possibilidade de recusa na participação, o que acarretaria em diferentes possibilidades de inclusão. Assim, a taxa de resposta $\left(t_{\text {resposta }}\right)$ em cada setor foi incorporada e a probabilidade final de inclusão de cada indivíduo foi obtida através da expressão $f=f_{1} \times f_{2} \times t_{\text {resposta }}$. Na área rural, a seleção dos indivíduos foi constituída por apenas um estágio, com isso a probabilidade de inclusão foi calculada através da expressão $f=f_{1} \mathrm{x}$ $t_{\text {resposta }}$, considerando $f_{1}=$ probabilidade de inclusão no primeiro estágio ( $\mathrm{n}^{\circ}$ de áreas rurais sorteados / no total de áreas rurais). Por fim, o peso de cada entrevistado foi obtido através do inverso da probabilidade de inclusão $(w=1 / f)$.

\section{Calibração dos examinadores, coleta e tabulação dos dados}

O processo de calibração foi conduzido por quatro instrutores, 33 examinadores (cirurgiõesdentistas) e 20 anotadores (acadêmicos do curso de Odontologia da Unimontes). As atividades foram desenvolvidas em cinco etapas: seleção dos voluntários, treinamento teórico, treinamento prático, coleta de dados para cálculo da concordância e cálculo da concordância. No cálculo da concordância, foi utilizado o coeficiente Kappa e foram considerados aptos para a coleta de dados somente aqueles examinadores que demonstraram níveis satisfatórios nos indicadores de concordância, além do acaso, isto é, kappa $>0,60^{17}$. Dos 33 examinadores treinados, 26 foram considerados aptos a participar da coleta.

Participaram da coleta de dados 24 examinadores e 20 anotadores previamente treinados e calibrados. Foram formadas 24 equipes de campo compostas por um examinador e um anotador.

Desenvolveu-se o Programa Coletor de Dados em Saúde (PCDS) ${ }^{18}$, para a coleta e a construção simultânea do banco de dados do projeto SB MOC. Esse programa foi instalado em um equipamento computacional móvel e portátil, um HP iPAQ Pocket PC, da série hx2000 (palmtop), e utilizado pelas equipes de campo.

Os dados coletados foram referentes às entrevistas e aos exames bucais realizados nos domicílios pertencentes aos setores e quadras previamente selecionados. Foram considerados como perda os casos de não localização do indivíduo, após três visitas ao domicílio.

Os exames intrabucais foram realizados em ambiente amplo sob iluminação natural, com espelho e sonda CPI da $\mathrm{OMS}^{14}$, adotando as normas de biossegurança preconizadas pelo Ministério da Saúde brasileiro ${ }^{4}$.

Ao final de cada dia de coleta das equipes de campo, os dados armazenados no computador de mão foram passados para um computador central. Ao término da coleta, os dados foram exportados para o programa PASW 17.0 e, em seguida, realizaram-se conferência, revisão e correção dos mesmos. 


\section{Variáveis do estudo}

Neste estudo foram considerados os seguintes grupos de variáveis:

1. Características demográficas e condição socioeconômica, constituídos pelas variáveis: localização geográfica, idade, sexo, cor de pele autodeclarada, estado conjugal, escolaridade, se estuda atualmente, tipo de escola, se trabalha atualmente, renda per capita mensal, moradia, $\mathrm{n}^{\circ}$ de residentes no domicílio, $\mathrm{n}^{\circ}$ de cômodos no domicílio e aglomeração do domicílio.

2. Uso de serviços odontológicos, constituído pelas variáveis: se fez uso de serviços odontológicos alguma vez na vida, tipo de serviço odontológico, tempo desde a última consulta ao dentista, motivo da consulta no último ano, se realiza visita periódica ao dentista, se recebeu informações sobre como evitar problemas bucais, se recebeu informações sobre como realizar autoexame de boca, e se recebeu orientação de higiene bucal.

3. Comportamentos relacionados à saúde, constituído pelas variáveis: frequência de escovação dental, uso de fio dental, uso de flúor tópico, hábitos bucais deletérios, realização de autoexame da boca, se é fumante, consumo de bebida alcoólica, prática atividade física.

4. Condições subjetivas de saúde bucal, constituída pelas variáveis: autopercepção da saúde bucal, da mastigação, da aparência dos dentes, da fala, do relacionamento afetado pela saúde bucal, da necessidade de tratamento dentário e dor nos dentes.

5. Condições objetivas de saúde bucal, constituída pelas variáveis: número de dentes cariados, número de dentes restaurados e sem cárie, número de dentes perdidos devido à cárie e índice CPO-D.

\section{Tratamento estatístico}

As variáveis categóricas foram descritas por meio de suas distribuições de frequências e as variáveis numéricas pelas medidas de tendência central e dispersão (média e erro-padrão), com correção pelo efeito do desenho (deff). A variável "número de dentes cariados (C)" foi dicotomizada para criação da variável desfecho presença de cárie (presente ou ausente). Aqueles indivíduos que apresentaram um ou mais dentes cariados ( $\mathrm{C}$ $\geq 1$ ) constituíram o grupo com cárie (cárie presente) e aqueles que apresentaram nenhum dente cariado $(\mathrm{C}=0)$, constituíram o grupo sem cárie (cárie ausente).

$\mathrm{Na}$ análise dos fatores associados à presença de cárie foi utilizado modelo de regressão logística hierarquizado ${ }^{19}$. Foram estimadas razões de chances (odds ratio) brutas e ajustadas, com seus respectivos intervalos de $95 \%$ de confiança.

Foi proposto um modelo de determinação da cárie dentária que contemplasse a autopercepção da saúde bucal, além dos determinantes sociais e de autocuidado. Uma adaptação do modelo sugerido pela $\mathrm{OMS}^{20}$ orientou a composição dos blocos de variáveis e a ordem de entrada dos mesmos. No modelo proposto, o nível distal foi constituído pelas variáveis do bloco, características demográficas e socioeconômicas; o nível intermediário, pelas variáveis do bloco uso de serviços odontológicos e comportamentos relacionados à saúde; e no nível proximal, incluíramse as variáveis do bloco condições subjetivas de saúde bucal (Figura 1).

Nesse modelo, hipotetizou-se que melhores condições socioeconômicas estariam associadas a melhor autocuidado com a saúde e maior uso dos serviços odontológicos. Considerou-se também que as variáveis do nível intermediário exerceram efeitos na autopercepção da saúde bucal,

\section{Nível distal}

Características demográficas Características socioeconômicas
Nível intermediário

Uso de serviços odontológicos

Comportamento relacionado à saúde

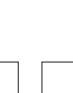$$
\text { sa }
$$

Condições subjetivas da saúde bucal (autopercepção)
Desfecho

Presença de cárie dentária 
isto é, quanto mais favorável as condições de uso dos serviços odontológicos e maiores autocuidados com a saúde, mais positiva seria a autopercepção das condições de saúde bucal, que por sua vez, estariam associadas às melhores condições relacionadas à cárie dentária. Um diferencial desse modelo, é que nele foram incluídas variáveis subjetivas (autopercepção) como previsoras de uma condição objetiva (cárie dentária).

Inicialmente, em cada bloco, foram realizadas análises bivariadas e as variáveis que apresentaram nível descritivo $\mathrm{p}<0,20$ nessa etapa foram selecionadas para a análise múltipla ${ }^{16}$. Para construção do modelo de regressão múltiplo de cada bloco adotou-se o procedimento passo à frente (stewise forward procedure). Permaneceram no modelo final aquelas variáveis que apresentaram associação significativa com o desfecho, ao nível de 0,05 .

As variáveis mantidas no modelo de regressão múltipla de cada bloco compuseram a análise hierarquizada. Os blocos das características demográficas e socioeconômicas foram os primeiros a serem incluídos no modelo, permanecendo como fator de ajuste para os determinantes intermediários e proximais. Em seguida, foram incluídas as variáveis do nível intermediário (uso de serviços odontológicos e comportamento relacionados à saúde), permanecendo no modelo somente aquelas que apresentaram nível descritivo $\mathrm{p}<0,05$, após o ajuste para as variáveis do nível distal. Por último, foi incluído o bloco composto pelas condições subjetivas de saúde bucal, permanecendo no modelo somente aquelas variáveis que apresentaram nível descritivo $\mathrm{p}<0,05$, após ajuste para as variáveis dos níveis distal e intermediário.

Em todas as etapas da modelagem, a significância dos coeficientes estimados foi verificada por meio do teste de Wald. Para avaliar a qualidade de ajuste dos modelos logísticos, utilizou-se o teste de Hosmer \& Lemeshow ${ }^{19}$. Todas as análises foram corrigidas pelo efeito do desenho e os programas estatísticos PASW ${ }^{\circledR} 17.0$ e STATA ${ }^{\circledR} 11.0$ foram utilizados para realização das mesmas.

\section{Resultados}

Foram identificados 834 adolescentes nos setores censitários, quadras e domicílios sorteados. Destes, 763 participaram do estudo, obtendo-se uma taxa de resposta de 91,5\%. O principal motivo das perdas foi a não localização dos indivíduos, após três visitas aos domicílios. A média da ida- de dos entrevistados foi de 17,1 anos, sendo que a maior parte era do sexo feminino (52,7\%). As distribuições dos adolescentes de acordo com as características demográficas e socioeconômicas, uso de serviços odontológicos, comportamento relacionado à saúde e condições subjetivas de saúde bucal estão apresentadas na Tabela 1 .

A maioria dos indivíduos examinados (71,3\%) apresentou experiência acumulada de cárie dentária (CPO-D $\geq 1)$, sendo que a condição mais prevalente foi a restauração $(\mathrm{O})$, com $55,6 \%$ da população apresentando pelo menos um dente restaurado. Os valores médios do CPO -D e seus componentes, bem como a proporção de contribuição de cada um deles no índice CPO -D estão apresentados na Tabela 2.

Com relação aos fatores associados à cárie dentária, a Tabela 3 apresenta as análises bivariadas e múltiplas dos três blocos de variáveis. As variáveis que não apresentaram associação estatisticamente significativa, ao nível de 0,20, na análise bivariada, não foram incluídas nesta tabela.

O modelo de regressão múltipla, ajustado pelas variáveis do bloco 1, apresentou bom ajuste $(\mathrm{p}=0,438)$ e revelou que as variáveis cor de pele e aglomeração do domicílio permaneceram associadas à cárie dentária. No bloco 2 , as seguintes variáveis foram significativamente associadas à cárie dentária, após o ajuste pelas demais variáveis do bloco: visita regular ao dentista, tipo de serviço odontológico, recebimento de informações sobre como evitar problemas bucais, consumo de cigarro e consumo de bebida alcoólica. $\mathrm{O}$ teste Hosmer \& Lemeshow sugeriu boa qualidade do modelo ajustado $(\mathrm{p}=0,728)$. O modelo de regressão múltipla, ajustado pelas variáveis do bloco 3 revelou que as variáveis autopercepção da saúde bucal e relato de dor nos dentes e/ou gengivas apresentaram associação significativa com a cárie dentária. Constatou-se boa qualidade desse modelo ajustado $(\mathrm{p}=0,969)$.

Os resultados da análise de regressão logística múltipla hierarquizada estão apresentados na Tabela 4. No bloco mais distal de determinação foram fatores associados à cárie: indivíduos que se autodeclararam de cor parda/preta/indígena e que moravam em domicílios com mais de uma pessoa por cômodo. No bloco intermediário, as chances de cárie foram maiores entre os indivíduos que utilizaram os serviços públicos/filantrópicos/nunca foram ao dentista, que não fizeram visitas regulares ao dentista, que fumavam e consumiam bebida alcoólica. No bloco proximal, verificou-se que aqueles que autoperceberam sua saúde bucal como péssima/ruim e regular, e que 
Tabela 1. Distribuição dos adolescentes de 15 a 19 anos segundo características demográficas e socioeconômicas, uso de serviços odontológicos, comportamento relacionado à saúde e condições subjetivas de saúde bucal. Montes Claros, MG, 2008/2009.

\begin{tabular}{|c|c|c|c|c|c|}
\hline Variáveis & $\mathbf{n}$ & $\%$ & Variáveis & $\mathbf{n}$ & $\%$ \\
\hline Características demográficas e socioecor & nicas & & Uso de serviços odontológicos & & \\
\hline Localização geográfica & & & Uso de serviços odontológicos & & \\
\hline Urbana & 754 & 99,6 & alguma vez na vida & & \\
\hline Rural & 09 & 0,4 & Nunca usou & 46 & 6,1 \\
\hline Idade & & & Usou & 717 & 93,9 \\
\hline 15 anos & 158 & 19,3 & Tipo de serviço odontológico & & \\
\hline 16 anos & 125 & 17,4 & Público/filantrópico & 366 & 55,6 \\
\hline 17 anos & 133 & 16,8 & Privado/plano de saúde/convênio & 351 & 44,4 \\
\hline 18 anos & 162 & 23,1 & Tempo desde a última consulta & & \\
\hline 19 anos & 185 & 23,4 & ao dentista & & \\
\hline Sexo & & & $\geq 1$ ano & 333 & 46,4 \\
\hline Masculino & 367 & 47,3 & $<1$ ano & 384 & 53,6 \\
\hline Feminino & 396 & 52,7 & Motivo da última visita ao dentista & & \\
\hline Cor de pele autodeclarada & & & Atendimento de rotina/manutenção & 416 & 59,7 \\
\hline Branca/amarela & 251 & 32,9 & Atendimento para tratamento & 298 & 40,3 \\
\hline Negra/índio/parda & 511 & 67,1 & Realiza visita periódica ao dentista & & \\
\hline Estado conjugal & & & Não & 529 & 73,8 \\
\hline Solteiro & 729 & 94,7 & $\operatorname{Sim}$ & 188 & 26,2 \\
\hline Casado & 34 & 5,3 & Recebeu informações sobre como & & \\
\hline Escolaridade & & & evitar problemas bucais & & \\
\hline Até 08 anos de estudo & 168 & 22,8 & Não & 203 & 26,6 \\
\hline 09 a 11 anos de estudo & 419 & 54,3 & Sim & 553 & 73,4 \\
\hline Mais de 11 anos de estudo & 176 & 22,9 & Recebeu informações sobre & & \\
\hline Estuda atualmente & & & como realizar autoexame da boca & & \\
\hline Não & 185 & 26,1 & Não & 618 & 80,1 \\
\hline Sim & 578 & 73,9 & Sim & 142 & 19,9 \\
\hline Tipo de escola & & & Recebeu orientação de higiene bucal & & \\
\hline Não estuda & 185 & 26,1 & Não & 117 & 14,9 \\
\hline Pública & 473 & 62,5 & Sim & 645 & 85,1 \\
\hline Privada & 105 & 11,4 & Comportamento relacionado à saúde & & \\
\hline Trabalha atualmente & & & Frequência de escovação dos dentes & & \\
\hline Sim & 173 & 24,5 & $<3$ vezes/dia & 217 & 28,9 \\
\hline Não & 590 & 75,5 & $\geq 3$ vezes/dia & 545 & 71,1 \\
\hline Renda per capita & & & Uso de fio dental & & \\
\hline Até R\$200,00 & 374 & 58,7 & Não usa & 426 & 58,8 \\
\hline Mais que $\mathrm{R} \$ 200,00$ & 309 & 41,3 & Usa & 336 & 41,2 \\
\hline Moradia & & & Uso de flúor tópico & & \\
\hline Alugada/cedida & 155 & 18,6 & Não & 509 & 60,7 \\
\hline Própria & 608 & 81,4 & Sim & 253 & 39,3 \\
\hline Número de residentes no domicílio & & & Hábitos bucais deletérios & & \\
\hline$>5$ pessoas & 175 & 26,2 & Sim & 302 & 41,2 \\
\hline$\leq 5$ pessoas & 588 & 73,8 & Não & 457 & 58,8 \\
\hline Número de cômodos no domicílio & & & Realização autoexame da boca & & \\
\hline$\leq 6$ cômodos & 295 & 36,9 & Não & 610 & 79,0 \\
\hline$>6$ cômodos & 468 & 63,1 & Sim & 152 & 21,0 \\
\hline Aglomeração do domicílio & & & Fumante & & \\
\hline$>1$ pessoa & 122 & 18,5 & Sim & 40 & 5,1 \\
\hline$\leq 1$ pessoa & 641 & 81,5 & Não & 723 & 94,9 \\
\hline
\end{tabular}

sentiram dor nos dentes ou gengivas nos últimos seis meses, tiveram maiores chances de cárie, após ajuste pelas variáveis dos blocos hierarquicamen- te superiores. O teste de Hosmer-Lemeshow mostrou boa qualidade de ajuste do modelo final ( $\mathrm{p}$ $=0,980$ ). 
Tabela 1. continuação

\begin{tabular}{|c|c|c|}
\hline Variáveis & n & $\%$ \\
\hline \multicolumn{3}{|l|}{ Consome bebida alcoólica } \\
\hline Sim & 146 & 18,8 \\
\hline Não & 617 & 81,2 \\
\hline \multicolumn{3}{|l|}{ Pratica de atividade física } \\
\hline Raramente/nunca & 260 & 31,8 \\
\hline Ocasionalmente & 160 & 20,0 \\
\hline Sempre/frequentemente & 343 & 48,2 \\
\hline \multicolumn{3}{|c|}{ Condições subjetivas de saúde bucal } \\
\hline \multicolumn{3}{|l|}{ Autopercepção da saúde bucal } \\
\hline Ótima ou boa & 505 & 65,6 \\
\hline Regular & 227 & 30,2 \\
\hline Ruim ou péssima & 30 & 4,2 \\
\hline \multicolumn{3}{|l|}{ Autopercepção da mastigação } \\
\hline Ótima ou boa & 600 & 78,0 \\
\hline Regular & 133 & 18,4 \\
\hline Ruim ou péssima & 29 & 3,6 \\
\hline \multicolumn{3}{|l|}{$\begin{array}{l}\text { Autopercepção da aparência } \\
\text { de dentes e/ou gengivas }\end{array}$} \\
\hline Ótima ou boa & 513 & 64,6 \\
\hline Regular & 192 & 26,5 \\
\hline Ruim ou péssima & 58 & 8,9 \\
\hline \multicolumn{3}{|c|}{$\begin{array}{l}\text { Autopercepção da fala devido aos } \\
\text { dentes e/ou gengivas }\end{array}$} \\
\hline Ótima ou boa & 671 & 86,0 \\
\hline Regular & 73 & 11,1 \\
\hline Ruim ou péssima & 19 & 2,9 \\
\hline \multicolumn{3}{|c|}{$\begin{array}{l}\text { Autopercepção do relacionamento } \\
\text { afetado pela saúde bucal }\end{array}$} \\
\hline Não afeta & 632 & 82,5 \\
\hline Afeta & 131 & 17,5 \\
\hline \multicolumn{3}{|l|}{$\begin{array}{l}\text { Autopercepção da necessidade } \\
\text { de tratamento dentário }\end{array}$} \\
\hline Não necessita & 266 & 34,9 \\
\hline Necessita & 494 & 65,1 \\
\hline \multicolumn{3}{|l|}{$\begin{array}{l}\text { Dor nos dentes e/ou gengivas } \\
\text { nos últimos seis meses }\end{array}$} \\
\hline Não & 510 & 67,2 \\
\hline Sim & 253 & 32,8 \\
\hline Total & 763 & 100,0 \\
\hline
\end{tabular}

\section{Discussão}

Constatou-se que a maioria dos adolescentes apresentava experiência de cárie dentária e que a chance dessa doença foi maior entre aqueles que se autodeclararam de cor parda/preta/indígena, aqueles que residiam em domicílios com maior aglomeração, os que nunca realizaram visitas regulares ao dentista, os que utilizaram serviços públicos ou filantrópicos, e/ou nunca haviam utilizado os serviços odontológicos, os fumantes,
Tabela 2. Prevalência e média (erro-padrão) do número de dentes cariados $(\mathrm{C})$, obturados $(\mathrm{O})$, perdidos $(\mathrm{P})$ e experiência de cárie (CPO-D) entre adolescentes de 15 a 19 anos. Montes Claros, MG, 2208/2009.

\begin{tabular}{lcccc}
\hline \multicolumn{1}{c}{ Variáveis } & $\mathbf{n}$ & $\%(\mathbf{I C 9 5} \%)$ & Média (E.P) & $\%$ \\
\hline Cariados (C) & & & $0,8(0,1)$ & 25,1 \\
$\mathrm{C}=0$ & 483 & $63,5(56,3-70,1)$ & & \\
$\mathrm{C} \geq 1$ & 280 & $36,5(29,4-43,7)$ & & \\
Obturados (O) & & & $2,4(0,2)$ & 70,1 \\
$\quad \mathrm{O}=0$ & 336 & $44,4(39,2-49,6)$ & & \\
$\mathrm{O} \geq 1$ & 427 & $55,6(50,4-60,8)$ & & \\
Perdidos (P) & & & $0,2(0,02)$ & 4,8 \\
$\mathrm{P}=0$ & 642 & $83,7(86,7-92,7)$ & & \\
$\mathrm{P} \geq 1$ & 121 & $16,3(12,4-21,0)$ & & \\
Índice CPO-D & & & $3,4(0,2)$ & 100,0 \\
CPO-D =0 & 214 & $28,7(25,5-32,3)$ & & \\
CPO-D $\geq 1$ & 549 & $71,3(67,7-74,5)$ & & \\
Total & 763 & 100,0 & &
\end{tabular}

IC95\%: intervalo de 95\% de confiança; E.P: erro-padrão da média.

os que consumiam bebida alcoólica, os que perceberam a sua saúde bucal como péssima/ruim e/ou regular, e entre aqueles que relataram ter sentido dor nos dentes ou gengivas nos últimos seis meses. Algumas dessas associações já haviam sido constadas em estudos prévios ${ }^{6,21-26}$. Por outro lado, são relativamente escassos na literatura estudos que adotaram modelos que consideram conjuntamente efeitos diretos e intermediários, pelos quais fatores sociais, comportamentais, uso de serviços e autopercepção, podem estar associados à saúde bucal dos adolescentes, embora alguns tenham sido propostos para adultos ${ }^{27}$.

$\mathrm{O}$ presente estudo revelou que a experiência de cárie (CPO-D $\geq 1$ ) acometeu $71,3 \%$ dos adolescentes. Esse percentual mostrou-se inferior aos encontrados no estado do Rio Grande do Sul $(92,7 \%)^{28}$, na cidade de Manaus $(88,8 \%)^{29}$, no Brasil $(76,1 \%)$ e na região Sudeste $(73,0 \%)$, no último levantamento nacional de Saúde Bucal $(2010)^{5}$. Ao se considerar estudos brasileiros, constata-se que a média do $\operatorname{CPOD}(3,4)$ registrada em Montes Claros foi inferior àquela registrada em 2002/2003 (6,2), assim como aquela registrada em $2010(4,3)$. Acredita-se que as melhores condições registradas entre os adolescentes do presente estudo sejam consequentes de políticas públicas de saúde bucal locais que beneficiaram este estrato etário. 
Tabela 3. Associação entre características demográficas, condição socioeconômica, uso de serviços odontológicos, comportamentos relacionados à saúde, condições subjetivas de saúde bucal e presença de cárie dentária: modelo de regressão logística múltipla não condicional. Montes claros, MG, 2008/2009.

\begin{tabular}{|c|c|c|c|c|c|c|c|c|}
\hline \multirow{3}{*}{ Variáveis } & \multicolumn{4}{|c|}{ Presença de cárie dentária } & \multirow[b]{3}{*}{$\mathrm{OR}^{\mathrm{a}}(\mathrm{IC} 95 \%)$} & \multirow[b]{3}{*}{$\mathrm{p}^{\mathrm{a}}$} & \multirow[b]{3}{*}{$\mathrm{OR}^{\mathrm{b}}(\mathrm{IC} 95 \%)$} & \multirow[b]{3}{*}{$\mathbf{p}^{\mathrm{b}}$} \\
\hline & \multicolumn{2}{|c|}{ Presente } & \multicolumn{2}{|c|}{ Ausente } & & & & \\
\hline & $\mathbf{n}$ & $\%$ & n & $\%$ & & & & \\
\hline \multirow{2}{*}{\multicolumn{9}{|c|}{$\begin{array}{l}\text { Bloco 1: Características } \\
\text { demográficas e socioeconômica } \\
\text { Cor de pele autodeclarada }\end{array}$}} \\
\hline & & & & & & & & \\
\hline Parda/Preta/Indígena & 203 & 40,3 & 308 & 59,7 & $1,7(1,1-2,5)$ & 0,019 & $1,6(1,1-2,4)$ & 0,028 \\
\hline Branca/Amarela & 76 & 28,9 & 175 & 71,1 & 1,0 & & 1,0 & \\
\hline \multicolumn{9}{|l|}{ Escolaridade } \\
\hline Até 8 anos & 78 & 46,3 & 90 & 53,7 & $1,6(1,1-2,4)$ & 0,046 & n.s & n.s \\
\hline De 9 a 11 anos & 143 & 32,9 & 276 & 67,1 & $0,9(0,6-1,3)$ & 0,567 & & \\
\hline Mais de 11 anos & 59 & 35,5 & 117 & 64,5 & 1,0 & & & \\
\hline \multicolumn{9}{|l|}{ Estuda atualmente } \\
\hline Não & 83 & 40,6 & 102 & 59,4 & $1,3(0,9-1,8)$ & 0,183 & n.s & n.s \\
\hline Sim & 197 & 35,1 & 381 & 64,9 & 1,0 & & & \\
\hline \multicolumn{9}{|l|}{ Tipo de escola } \\
\hline Não estuda & 83 & 40,6 & 102 & 59,4 & $2,5(1,2-5,3)$ & 0,020 & n.s & n.s \\
\hline Pública & 175 & 37,5 & 298 & 62,5 & $2,2(1,1-4,3)$ & 0,024 & & \\
\hline Privada & 22 & 21,5 & 83 & 78,5 & 1,0 & & & \\
\hline \multicolumn{9}{|l|}{ Trabalha atualmente } \\
\hline Sim & 78 & 42,2 & 95 & 57,8 & $1,4(0,9-2,0)$ & 0,105 & n.s & n.s \\
\hline Não & 202 & 34,7 & 388 & 65,3 & 1,0 & & & \\
\hline \multicolumn{9}{|l|}{ Aglomeração do domicílio } \\
\hline$>1$ pessoa & 70 & 44,1 & 52 & 55,9 & $2,5(1,5-4,2)$ & & $2,4(1,5-4,0)$ & 0,001 \\
\hline \multirow[t]{2}{*}{$\leq 1$ pessoa } & 210 & 33,8 & 431 & 66,2 & 1,0 & & 1,0 & \\
\hline & & & & & & & \multicolumn{2}{|c|}{$\chi_{\mathrm{HL}}^{2}(2)=1,65 ; \mathrm{p}=0,438$} \\
\hline
\end{tabular}

Foi observado que $36,5 \%(29,4 \%-43,7 \%)$ dos adolescentes apresentaram pelo menos um dente com cárie $(C \geq 1)$; esse resultado foi inferior aos encontrados em estudos prévios entre adolescentes brasileiros ${ }^{6,20}$, cujos percentuais variaram de $53,3 \%$ a $90,3 \%$. Esses achados sugerem que o acesso ao flúor, dentre outros determinantes da cárie, foi desigual nessas populações. Por outro lado, é perceptível que a prevalência da cárie dentária tem-se reduzido entre os adolescentes brasileiros, como comprovam as pesquisas epidemiológicas nacionais realizadas em $1986^{3}$, em $2002 / 2003^{4}$, e em $2010^{5}$.

Verificou-se também que os adolescentes apresentaram em média 2,4 dentes restaurados e que essa condição (componente $\mathrm{O}$ ) correspondeu a $70,1 \%$ da composição do índice CPO-D, superior aos percentuais observados no Brasil $(50,8 \%)^{5}$ e no Peru $(63,5 \%)^{30}$. Uma maior proporção do componente $\mathrm{O}$ na composição do índice CPO-D indica menores proporções dos componentes $\mathrm{C}$ (dentes cariados) e $\mathrm{P}$ (dentes perdidos) e, consequentemente, melhor condição de saúde bucal no que se refere à cárie dentária. Esse resultado pode ser reflexo de um acesso satisfatório dessa população aos serviços odontológicos, uma vez que conforme resultados de dois inquéritos nacionais brasileiros houve uma maior frequência de uso dos serviços odontológicos em $2010^{5}$ do que em 2002/2003 ${ }^{17}$. Na população investigada essa situação fica ainda mais evidente visto que mais de $90 \%$ dos entrevistados relataram ter ido ao dentista alguma vez na vida.

Apesar de ser um problema de saúde pública, a perda dentária tem sido pouco investigada entre adolescentes brasileiros. No presente estudo, foi constatado que $83,7 \%$ dos adolescentes apresentaram todos os dentes presentes na boca, esse percentual ainda não atingiu a meta estabelecida pela OMS para o ano de 2000, para jovens com 18 anos de idade, isto é, $85 \%$ dos indivíduos com todos os dentes presentes na boca. Nesse 
Tabela 3. continuação

\begin{tabular}{|c|c|c|c|c|c|c|c|c|}
\hline \multirow{3}{*}{ Variáveis } & \multicolumn{4}{|c|}{ Presença de cárie dentária } & \multirow[b]{3}{*}{$\mathrm{OR}^{\mathrm{a}}(\mathrm{IC} 95 \%)$} & \multirow[b]{3}{*}{$\mathbf{p}^{\mathrm{a}}$} & \multirow[b]{3}{*}{$\mathrm{OR}^{\mathrm{b}}(\mathrm{IC} 95 \%)$} & \multirow[b]{3}{*}{$\mathbf{p}^{\mathrm{b}}$} \\
\hline & \multicolumn{2}{|c|}{ Presente } & \multicolumn{2}{|c|}{ Ausente } & & & & \\
\hline & $\mathbf{n}$ & $\%$ & $\mathbf{n}$ & $\%$ & & & & \\
\hline \multicolumn{9}{|l|}{ Blocos 2 e 3: Uso de } \\
\hline \multicolumn{9}{|l|}{ serviços odontológicos e } \\
\hline \multicolumn{9}{|l|}{ comportamento relacionado } \\
\hline \multicolumn{9}{|l|}{ à saúde } \\
\hline \multicolumn{9}{|l|}{ Tipo de serviço odontológico } \\
\hline $\begin{array}{l}\text { Público/filantrópico/ } \\
\text { nunca foi ao dentista }\end{array}$ & 192 & 44,4 & 220 & 55,6 & $2,3(1,6-3,5)$ & 0,000 & $2,1(1,3-3,4)$ & 0,004 \\
\hline $\begin{array}{l}\text { Privado/plano de saúde/ } \\
\text { convênio }\end{array}$ & 88 & 25,5 & 263 & 74,5 & 1,0 & & 1,0 & \\
\hline \multicolumn{9}{|l|}{$\begin{array}{l}\text { Tempo desde a última } \\
\text { consulta ao dentista }\end{array}$} \\
\hline Nunca foi ao dentista & 19 & 39,6 & 27 & 60,4 & $1,6(0,8-2,9)$ & 0,153 & n.s & n.s \\
\hline$\geq 1$ ano & 147 & 44,1 & 186 & 55,9 & $1,9(0,4-2,6)$ & 0,000 & & \\
\hline$<1$ ano & 114 & 29,6 & 270 & 70,4 & 1,0 & & & \\
\hline \multicolumn{9}{|l|}{$\begin{array}{l}\text { Realiza visita regular ao } \\
\text { dentista }\end{array}$} \\
\hline Não/nunca foi ao dentista & 239 & 41,0 & 336 & 59,0 & $2,4(1,4-4,0)$ & 0,002 & $1,9(1,1-3,5)$ & 0,029 \\
\hline Sim & 41 & 22,7 & 147 & 77,3 & 1,0 & & 1,0 & \\
\hline \multicolumn{9}{|l|}{$\begin{array}{l}\text { Recebeu informações sobre } \\
\text { como evitar problemas } \\
\text { bucais }\end{array}$} \\
\hline Não/nunca foi ao dentista & 91 & 45,0 & 112 & 55,0 & $1,6(0,9-2,7)$ & 0,057 & $1,7(1,1-2,6)$ & 0,023 \\
\hline Sim & 186 & 33,3 & 367 & 66,7 & 1,0 & & 1,0 & \\
\hline \multicolumn{9}{|l|}{$\begin{array}{l}\text { Frequência de escovação } \\
\text { dos dental }\end{array}$} \\
\hline$<3$ vezes/dia & 90 & 40,6 & 127 & 59,4 & $1,3(0,8-1,9)$ & 0,200 & n.s & n.s \\
\hline$\geq 3$ vezes/dia & 190 & 34,9 & 355 & 65,1 & 1,0 & & & \\
\hline \multicolumn{9}{|l|}{ Uso de fio dental } \\
\hline Não usa & 182 & 40,7 & 244 & 59,3 & $1,6(1,1-2,3)$ & 0,034 & n.s & n.s \\
\hline Usa & 98 & 30,7 & 238 & 69,3 & 1,0 & & & \\
\hline \multicolumn{9}{|l|}{ Fumante } \\
\hline Sim & 29 & 67,4 & 11 & 32,6 & $3,9(2,2-6,8)$ & 0,000 & $3,5(1,8-6,9)$ & 0,001 \\
\hline Não & 251 & 34,9 & 472 & 65,1 & 1,0 & & 1,0 & \\
\hline \multicolumn{9}{|l|}{ Consome bebida alcoólica } \\
\hline Sim & 72 & 49,1 & 74 & 50,9 & $1,1(1,4-2,7)$ & 0,001 & $1,8(1,1-2,8)$ & 0,014 \\
\hline \multirow[t]{2}{*}{ Não } & 208 & 33,6 & 409 & 66,4 & 1,0 & & 1,0 & \\
\hline & & & & & & & \multicolumn{2}{|c|}{$\chi_{\mathrm{HL}}^{2}(5)=2,82 ; \mathrm{p}=0,728$} \\
\hline
\end{tabular}

sentido, considerando que no ano da realização desse estudo (2008/2009) a meta proposta para 2000 ainda não fora totalmente atingida e, que, durante o $4^{\circ}$ Congresso Mundial de Odontologia Preventiva em 1993, a OMS propôs nova meta para o ano 2010: não haver perda dentária aos 18 anos, conclui-se que a condição de saúde bucal da população investigada ainda está aquém de uma condição adequada. Por outro lado, quando se considera o cenário nacional, a situação do adolescente do município em estudo (Montes Claros-MG) é mais favorável, uma vez que essa condição (componente P) contribuiu com 4,8\% na composição do CPO-D, percentual inferior aos observados entre adolescentes de todas as regiões brasileiras, cujos valores variaram de 5,2\% (região Sul) a $16,8 \%$ (região Norte) $^{5}$.

Com relação ao modelo estatístico adotado, ressalta-se a utilização de modelos hierarquizados que permitiram identificar os possíveis 
Tabela 3. continuação

\begin{tabular}{|c|c|c|c|c|c|c|c|c|}
\hline \multirow{3}{*}{ Variáveis } & \multicolumn{4}{|c|}{ Presença de cárie dentária } & \multirow[b]{3}{*}{$\mathrm{OR}^{\mathrm{a}}(\mathrm{IC} 95 \%)$} & \multirow[b]{3}{*}{$\mathrm{p}^{\mathrm{a}}$} & \multirow[b]{3}{*}{$\mathrm{OR}^{\mathrm{b}}(\mathrm{IC} 95 \%)$} & \multirow[b]{3}{*}{$\mathbf{p}^{\mathrm{b}}$} \\
\hline & \multicolumn{2}{|c|}{ Presente } & \multicolumn{2}{|c|}{ Ausente } & & & & \\
\hline & $\mathbf{n}$ & $\%$ & $\mathbf{n}$ & $\%$ & & & & \\
\hline \multicolumn{9}{|c|}{$\begin{array}{l}\text { Bloco 3: Condições subjetivas } \\
\text { de saúde bucal } \\
\text { Autopercepção da saúde } \\
\text { bucal }\end{array}$} \\
\hline Péssima/ruim & 23 & 71,0 & 07 & 29,0 & $6,1(2,2-16,7)$ & 0,001 & $3,7(1,3-10,2)$ & 0,001 \\
\hline Regular & 114 & 48,5 & 113 & 51,5 & $2,3(1,6-3,4)$ & 0,000 & $1,8(1,3-2,7)$ & 0,002 \\
\hline Ótima/boa & 142 & 28,7 & 363 & 71,3 & 1,0 & & 1,0 & \\
\hline \multicolumn{9}{|l|}{$\begin{array}{l}\text { Autopercepção da } \\
\text { mastigação }\end{array}$} \\
\hline Péssima/ruim & 16 & 59,5 & 13 & 40,5 & $2,9(1,3-6,7)$ & 0,009 & n.s & n.s \\
\hline Regular & 65 & 46,5 & 68 & 53,5 & $1,8(1,1-2,8)$ & 0,020 & & \\
\hline Ótima/boa & 198 & 33,0 & 402 & 67,0 & 1,0 & & & \\
\hline \multicolumn{9}{|c|}{$\begin{array}{l}\text { Autopercepção da aparência } \\
\text { de dentes e/ou gengivas }\end{array}$} \\
\hline Péssima/ruim & 35 & 57,9 & 23 & 42,1 & $3,2(1,6-6,2)$ & 0,001 & n.s & n.s \\
\hline Regular & 89 & 44,9 & 103 & 55,1 & $1,9(1,1-3,4)$ & 0,039 & & \\
\hline Ótima/boa & 156 & 30,2 & 357 & 69,8 & 1,0 & & & \\
\hline \multicolumn{9}{|c|}{$\begin{array}{l}\text { Autopercepção da fala } \\
\text { devido aos dentes e/ou } \\
\text { gengivas }\end{array}$} \\
\hline Péssima/ruim & 10 & 53,4 & 09 & 46,6 & $2,1(0,8-5,5)$ & 0,118 & n.s & n.s \\
\hline Regular & 33 & 42,6 & 40 & 57,4 & $1,4(0,8-2,3)$ & 0,205 & & \\
\hline Ótima/boa & 237 & 35,1 & 434 & 64,9 & 1,0 & & & \\
\hline \multicolumn{9}{|c|}{$\begin{array}{l}\text { Autopercepção do } \\
\text { relacionamento afetado pela } \\
\text { saúde bucal }\end{array}$} \\
\hline Afeta & 66 & 48,3 & 65 & 51,7 & $1,8(1,3-2,6)$ & 0,002 & n.s & n.s \\
\hline Não afeta & 214 & 34,0 & 418 & 66,0 & 1,0 & & & \\
\hline \multicolumn{9}{|c|}{$\begin{array}{l}\text { Dor nos dentes e/ou gengiva } \\
\text { nos últimos seis meses }\end{array}$} \\
\hline Sim & 130 & 48,6 & 123 & 51,4 & $2,1(1,4-3,3)$ & 0,001 & $1,4(1,1-2,5)$ & 0,019 \\
\hline Não & 150 & 30,6 & 360 & 69,4 & 1,0 & & 1,0 & \\
\hline & & & & & & & $\mathrm{HL}(2)=0,062$; & $=0,969$ \\
\hline
\end{tabular}

$\mathrm{OR}^{\mathrm{a}}$ : odds ratio - valores brutos e $\mathrm{OR}^{\mathrm{b}}$ : odds ratio - valores ajustados pelas outras variáveis do bloco; $\chi_{\mathrm{HL}}^{2}$ : teste Hosmer \& Lemeshow. n.s: não significativa.

fatores associados à cárie dentária de forma independente, seguindo níveis hierárquicos de determinação. O modelo estimado revelou que, no nível distal, a chance mais elevada de cárie entre aqueles que se declararam cor de pele parda/preta/indígena é compatível com resultado de outro estudo $^{6}$ e tem sido atribuída à pior condição socioeconômica destes grupos étnicos em relação aos brancos na sociedade brasileira. Em concordância com estudo anterior ${ }^{23}$, morar em residências com maior número de pessoas por cômodo também foi considerado um fator significantemente associado à cárie dentária. Nessa situação, a superlotação no domicílio é um indicador de condição socioeconômica mais baixa, que por sua vez está associada às piores condições de saú$\mathrm{de}^{31}$. A variável renda per capita não apresentou associação com a cárie na análise bruta e por isso não foi incluída no modelo múltiplo, acredita-se que seja devido às perdas de informações dessa variável, uma vez que mais de $10 \%$ dos adolescentes não informaram a renda per capita da família. Provavelmente a magnitude das associações entre as variáveis cor de pele e aglomeração do domicílio com a cárie poderia ser modificada na presença da variável renda per capita. 
Tabela 4. Resultados da análise de regressão logística múltipla não condicional hierarquizada para presença de cárie dentária. Montes Claros, MG, 2008/2009.

\begin{tabular}{|c|c|c|c|}
\hline Variáveis & $\beta(\mathbf{E P})$ & $\mathrm{OR}^{\mathrm{a}}(\mathrm{IC}$ 95\%) & $\mathrm{p}^{\mathrm{a}}$ \\
\hline \multicolumn{4}{|c|}{ Bloco 1: Características demográficas e condição socioeconômica } \\
\hline \multicolumn{4}{|l|}{ Cor de pele autodeclarada } \\
\hline Parda/negra/outras & $0,5(0,2)$ & $1,6(1,1-2,4)$ & 0,028 \\
\hline Branca/amarela & & 1,0 & \\
\hline \multicolumn{4}{|l|}{ Aglomeração do domicílio } \\
\hline$>1$ pessoa & $0,9(0,3)$ & $2,4(1,5-4,0)$ & 0,001 \\
\hline$\leq 1$ pessoa & & 1,0 & \\
\hline \multicolumn{4}{|l|}{ Blocos 2 e 3:Uso de serviços odontológicos } \\
\hline \multicolumn{4}{|l|}{ e comportamentos relacionados à saúde } \\
\hline \multicolumn{4}{|l|}{ Realiza visita regular ao dentista } \\
\hline Não/nunca foi ao dentista & $0,6(0,3)$ & $1,9(1,1-3,3)$ & 0,040 \\
\hline Sim & & 1,0 & \\
\hline \multicolumn{4}{|l|}{ Tipo de serviço odontológico } \\
\hline Público/filantrópico/nunca foi ao dentista & $0,6(0,3)$ & $1,8(11-3,0)$ & 0,019 \\
\hline Privado/plano de saúde/convênio & & 1,0 & \\
\hline \multicolumn{4}{|l|}{ Fumante } \\
\hline Sim & $1,4(0,3)$ & $4,1(2,2-7,7)$ & 0,000 \\
\hline Não & & 1,0 & \\
\hline \multicolumn{4}{|l|}{ Consome bebida alcoólica } \\
\hline Sim & $0,6(0,2)$ & $1,8(1,1-2,8)$ & 0,024 \\
\hline Não & & 1,0 & \\
\hline \multicolumn{4}{|l|}{ Bloco 4: Condições subjetivas de saúde bucal } \\
\hline \multicolumn{4}{|l|}{ Autopercepcão da saúde bucal } \\
\hline Péssima/ruim & $1,8(0,5)$ & $5,9(2,1-16,8)$ & 0,001 \\
\hline Regular & $0,6(0,2)$ & $1,9(1,4-2,6)$ & 0,031 \\
\hline Ótima/boa & & 1,0 & \\
\hline \multicolumn{4}{|l|}{ Dor nos dentes e/ou gengivas nos últimos 6 meses } \\
\hline Sim & $0,7(0,2)$ & $2,0(1,3-3,0)$ & 0,004 \\
\hline \multirow[t]{2}{*}{ Não } & & 1,0 & \\
\hline & & \multicolumn{2}{|c|}{$\chi_{\mathrm{HL}}^{2}(8)=2,03 ; \mathrm{p}=0,980$} \\
\hline
\end{tabular}

$\mathrm{OR}^{\mathrm{a}}$ : odds ratio - valores ajustados pelas variáveis internas do bloco e pelo $(\mathrm{s})$ bloco $(\mathrm{s})$ superior; $\beta(\mathrm{EP})=$ coeficiente estimado (erro-padrão); $\chi_{H}^{2}$ : teste Hosmer -Lemeshow.

No nível intermediário constatou-se que as chances de cárie entre os indivíduos que não utilizaram regularmente os serviços odontológicos foram 1,9 vezes àquelas observadas entre os que faziam uso regular desses serviços. De maneira semelhante, outros estudos também encontraram associação entre o uso dos serviços odontológicos e a cárie dentária ${ }^{20,22}$. Essa associação já era esperada, uma vez que indivíduos que usam regularmente os serviços odontológicos apresentam maior possibilidade de acesso a procedimentos de manutenção da saúde bucal, prevenção de doenças e tratamento precoce. Por outro lado, a variável acesso a informações sobre como evitar problemas bucais apresentou associação significativa com a cárie dentária $(\mathrm{OR}=1,7)$ na análise múltipla dentro do bloco, mas na análise hierar- quizada, quando ajustada pelas variáveis do bloco distal, essa variável não mostrou associação com o desfecho. Nesse contexto, esse resultado é surpreendente, parece que o acesso à informação per se não garante que o indivíduo adquira e coloque em prática os hábitos e cuidados positivos com a sua saúde bucal, especialmente na adolescência, que é considerada um período de maior comportamento de risco para cárie dentária ${ }^{32}$.

Foi constatado também que os adolescentes usuários de serviços públicos ou filantrópicos apresentaram maiores chances de cárie dentária quando comparados com aqueles que utilizam os serviços privados/convênios/planos de saúde, corroborando com os achados de outro estudo ${ }^{33}$. Geralmente, os usuários do serviço público pertencem a uma população economicamente mais 
pobre e com menor acesso ao atendimento odontológico, o que explicaria a associação encontrada. Esse fato sugere a necessidade de garantir a prevenção e controle da cárie e o acesso a tratamentos dentários restauradores no serviço público.

Os adolescentes que possuíam o hábito de fumar apresentaram maiores chances de cárie (OR $=4,1$ ), em consonância com estudo prévio ${ }^{34}$. Constatou-se também uma associação significativa entre a cárie e o consumo de bebida alcoólica $(\mathrm{OR}=1,8)$. Não foram identificados outros estudos que avaliaram essa associação entre adolescentes. Por outro lado, verificou-se previamente, entre adolescentes brasileiros, que o consumo de tabaco e de bebida alcoólica estão associados à dor dentária ${ }^{35}$, e que o tabagismo está associado à perda dentária ${ }^{36}$. Outro estudo demonstrou que a cárie está positivamente associada à dor de origem dentária e que aquela é a principal causa de perdas dentárias ${ }^{37}$. Ademais, um estudo constatou que ex-fumantes e não fumantes apresentaram maior autocuidado com a saúde bucal do que fumantes ${ }^{34}$. Petersen e Ogawa ${ }^{38}$ sugerem que os indivíduos fumantes e os que consomem bebidas alcoólicas estão predispostos à cárie, periodontite e até mesmo ao câncer de boca.

No bloco proximal, após ajuste pelas variáveis dos blocos intermediário e distal, foi identificada associação entre cárie e autopercepção da saúde bucal. A prevalência de cárie foi maior entre os adolescentes que avaliaram negativamente sua saúde bucal, corroborando com outro estudo9. Sendo a cárie uma das principais causas de dor dentária ${ }^{37}$, é compreensível que a associação com a autopercepção da saúde bucal seja positiva, uma vez que as pessoas percebem sua condição bucal a partir dos sintomas e dos problemas funcionais e sociais decorrentes das doenças bu- cais $^{39}$. A percepção da condição de saúde bucal é considerada como uma importante medida para a Odontologia, por estar associada à predisposição dos indivíduos ao uso dos serviços de saúde e à condição clínica presente ${ }^{23}$. Num estudo em Pelotas-RS constatou-se que os adolescentes com melhor autopercepção da saúde bucal consultaram mais do que aqueles com autopercepção negativa, e aqueles que perceberam algum problema odontológico utilizaram $40 \%$ a mais os serviços quando comparados com aqueles que não perceberam ${ }^{40}$. Na presente pesquisa também foi verificada associação positiva entre cárie e dor nos dentes/gengivas, concordando com estudo prévio $^{37}$. A chance de cárie dentária foi maior entre os indivíduos que sentiram dor nos dentes/ gengivas nos últimos seis meses $(\mathrm{OR}=2,0)$, fato já esperado, pois a cárie é considerada uma das principais causas da dor de origem dentária ${ }^{34}$.

Os resultados desse estudo revelaram que a cárie dentária está associada aos fatores comportamentais e de autocuidado, bem como à autopercepção da saúde bucal, mediados por condições socioeconômicas. Recomenda-se o desenvolvimento de intervenções planejadas para promover a saúde bucal e mudança de comportamento dessa população, direcionadas especialmente aos grupos populacionais que apresentaram polarização da doença. A higiene bucal, fluoração e alimentação não cariogênica constituem medidas eficazes para enfrentar os problemas bucais, que para terem êxito precisam fundamentar-se em programas educativos ${ }^{41}$. Nesse sentido, a educação em saúde representa uma importante possibilidade de ampliar a atuação das práticas de promoção da saúde bucal no espaço público ${ }^{41}$, e o ambiente escolar pode ser um espaço promotor da saúde bucal de adolescentes. 


\section{Colaboradores}

MF Silveira e AMEBL Martins participaram de todas as etapas: concepção, delineamento, análise e interpretação dos dados, redação do artigo. RS Freire e MO Nepomuceno contribuíram na análise e interpretação dos dados e redação do artigo. LF Marcopito orientou todas as fases do estudo.

\section{Referências}

1. Freire MCM, Reis SCGB, Figueiredo N, Peres KG, Moreira RS, Antunes JLF. Determinantes individuais e contextuais da cárie em crianças brasileira de 12 anos em 2010. Rev Saude Publica 2013; 47(Supl. 3):40-49.

2. Narvai PC, Frazão P. Saúde bucal no Brasil: muito além do céu da boca. Rio de Janeiro: Editora Fiocruz; 2008.

3. Brasil. Ministério da Saúde (MS). Levantamento epidemiológico em saúde bucal: Brasil, zona urbana, 1986. Brasília: MS; 1988.

4. Brasil. Ministério da Saúde (MS). Secretaria de atenção à saúde. Secretaria de vigilância em saúde. Departamento de atenção básica. Coordenação geral de saúde bucal. Projeto SB Brasil 2003 - Condições de saúde bucal da população brasileira 2002-2003: resultados principais. Brasília: MS; 2004.

5. Brasil. Ministério da Saúde (MS). Secretaria de atenção à saúde. Secretaria de vigilância em saúde. Departamento de atenção básica. Coordenação geral de saúde bucal. SB Brasil 2010 - Pesquisa nacional de saúde bucal: resultados principais. Brasília: MS; 2011.

6. Frias AC, Antunes JLF, Junqueira SR, Narvai PC. Determinantes individuais e contextuais da prevalência de cárie dentária não tratada no Brasil. Rev Panam Salud Publica 2007; 22(4):279-285.

7. Costa SM, Abreu MHNG, Vasconcelos M, Lima RCGS, Verdi M, Ferreira EF. Desigualdades na distribuição da cárie dentária no Brasil: uma abordagem bioética. Cien Saude Colet 2013; 18(2):461-470.

8. Peres SHCS, Carvalho FS, Carvalho CP,Bastos JRM, Lauris JRP. Polarização da cárie dentária em adolescentes, na região sudoeste do estado de São Paulo, Brasil. Cien Saude Colet 2008; 13(Supl. 2):2155-2162.

9. Lopes MC, Silva PR, Biazevic MGH, Rebelo MAB, Crosato EM. Necessidade de Tratamento Decorrente da Cárie Dentária em Estudantes de 15 a 19 Anos de Idade, em Manaus-AM, Brasil e fatores associados. Pesquisa Brasileira em Odontopediatria e Clínica Integrada 2012; 12(1):83-88.

10. Santos NCN, Alves TDB, Freitas VS, Jamelli SR, Sarinho ESC. A saúde bucal de adolescentes: aspctos d higiene, de cárie dentária e doença periodontal nas cidades de Recife, Pernambuco e Feira de Santana, Bahia. Cien Saude Colet 2007; 12(5):1155-1166.

11. Garbin CAS, Garbin AJI, Arcieri RM, Saliba NA, Gonçalves PE. La Salud Bucal en la Percepción Del Adolescente. Revista salud pública 2009; 11(2):268-277.

12. Ruzany MH, Szwarcwald CL. Oportunidades perdidas de atenção integral ao adolescente: resultado de estudo piloto. Rev Adolescencia Latino americana 2000; 2(1):26-35.

13. Barbosa TS, Mialhe FL, Castilho ARF, Gavião MBD. Qualidade de vida e saúde bucal. Physis 2010; 20(1): 283-300.

14. World Health Organization (WHO). Oral health surveys: basic methods. $4^{\text {th }}$ ed. Geneva: WHO; 1997.

15. Roncalli AG, Frazão P, Pattussi MP, Araújo IC, Ely HC, Batista SM. Projeto SB2000: uma perspectiva para a consolidação da Epidemiologia em Saúde Bucal Coletiva. Rev Brasileira de Odontologia em Saúde Coletiva 2000; 1(2):9-25.

16. Szwarcwald CL, Damacena GN. Amostras complexas em inquéritos populacionais: planejamento e implicações na análise estatística dos dados. Rev Brasileira de Epidemiologia 2008; 11(1):38-45. 
17. Bartko JJ. The intraclass correlation coefficient as a measure of reliability. Psychol Rep 1966; (19):3-11.

18. Martins AMEBL, Rodrigues CAQ, Haikal DS, Silveira MF, Mendes DC, Oliveira MP, Andrade AF, Freitas CV. Desenvolvimento de um programa de computador para levantamentos epidemiológicos sobre condições de saúde bucal. Revista Unimontes Científica 2012; 14(1):30-42.

19. Hosmer DW, Lemeshow S. Applied logistic regression. 2a ed. New York: John Wiley \& Sons; 2000.

20. Petersen PE. The World Oral Health Report 2003: continuous improvement of oral health in the $21^{\text {st }}$ century - the approach of the WHO Global Oral Health Programme. Community Dent Oral Epidemiol 2003; 31(Supl. 1):3-24.

21. Lopes MC, Silva PR, Biazevic MGH, Rebelo MAB, Crosato EM. Necessidade de Tratamento Decorrente da Cárie Dentária em Estudantes de 15 a 19 Anos de Idade, em Manaus-AM, Brasil e fatores associados. Pesquisa Brasileira em Odontopediatria e Clínica Integrada 2012; 12(1):83-88.

22. Loyola APP, Solis CEM, Yãnez AB Marin NP, Marquez AI, Maupome G. Prevalence and severity of dental caries in adolescents aged 12 and 15 living in communities with various fluoride concentrations. J Public Health Dent 2007; 67(1):8-13.

23. Lacerda JT, Castilho EA, Calvo MCM, Freitas SFT. Saúde bucal e o desempenho diário de adultos em Chapecó, Santa Catarina, Brasil. Cad Saude Publica 2008; 24(8):1846-1858.

24. Gushi LL, Soares MC, Forni TIB, Vieira V, Wada RS, Sousa MLR. Cárie dentária em adolescentes de 15 a 19 anos de idade no Estado de São Paulo, Brasil, 2002. Cad Saude Publica 2005; 21(5):1383-1391.

25. Tada A, Hanada N. Sexual differences in smoking behavior and dental caries experience in young adults. $P u$ blic Health 2002; 116(6):341-346.

26. Borges CM, Cascaes AM, Fischer TK, Boing AF, Peres MA, Peres KG. Dor nos dentes e gengivas e fatores associados em adolescentes brasileiros: análise do inquérito nacional de saúde bucal SB - Brasil 2002-2003. Cad Saude Publica 2008; 24(8):1825-1834.

27. Baker SR. Applying Andersen's behavioural model to oral health: what are the contextual factors shaping perceived oral health outcomes? Community Dent Oral Epidemiol 2009; 37(6):485-494.

28. Celeste RK, Nadanovsky P, De Leon AP. Associação entre procedimentos preventivos no serviço público de odontologia e a prevalência de cárie dentária. Rev Saúde Pública 2007; 41(5):830-838.

29. Viana ARP, Parente RCP, Borras MR, Rebelo MAB. Prevalência de cárie dentária e condições socioeconômicas em jovens alistandos de Manaus, Amazonas, Brasil. Rev Brasileira de Epidemiologia 2009; 12(4):680-687.
30. Loyola MC. Características socioeconómicas y salud bucal de escolares de instituciones educativas públicas. Kiru 2009; 6(2):78-80.

31. Antunes JLF, Narvai PC, Nugent ZJ. Inequalities in the distribution of dental caries. Community Dent Oral Epidemiol 2004; 32(1):41-48.

32. Elias MS, Cano MAT, Junior WM, Ferriani MGC. A importância da saúde bucal para adolescentes de diferentes estratos Sociais do município de Ribeirão Preto. Revista latino-americana de Enfermagem 2001; 9(1):8895.

33. Gushi LL, Soares MC, Forni TIB, Vieira V, Wada RS, Sousa MLR. Cárie dentária em adolescentes de 15 a 19 anos de idade no Estado de São Paulo, Brasil, 2002. Cad Saude Publica 2005; 21(5):1383-1391.

34. Tada A, Hanada N. Sexual differences in smoking behavior and dental caries experience in young adults. $\mathrm{Pu}$ blic Health 2002; 116(6):341-346.

35. Peres MA, Iser BPM, Peres KG, Malta DC, Antunes JLF. Desigualdades contextuais e individuais da prevalência de dor dentária em adultos e idosos no Brasil. Cad Saude Publica 2012; 28(Sup: S) 114-123.

36. Ojima M, Hanioka T, Tanaka Keiko, Aoyama H. Cigarette smoking and tooth loss experience among young adults: a national record linkage study. BMC Public Health 2007; 7:313-320.

37. Borges CM, Cascaes AM, Fischer TK, Boing AF, Peres MA, Peres KG. Dor nos dentes e gengivas e fatores associados em adolescentes brasileiros: análise do inquérito nacional de saúde bucal SB- Brasil 2002-2003. Cad Saude Publica 2008; 24(8):1825-1834.

38. Petersen PE, Ogawa H. Strengthening the prevention of periodontal disease: the WHO approach. $J$ Periodontal 2005; 76(12):2187-2193.

39. Reisine ST, Bailit HL. Clinical oral health status and adult perceptions of oral health. Soc Sci Med Med Psychol Med Sociol 1980; 14A(6):597-605.

40. Araújo CS, Lima RC, Peres MA, Barros AJD. Utilização de serviços odontológicos e fatores associados: um estudo de base populacional no Sul do Brasil. Cad Saude Publica 2009; 25(5):1063-1072.

41. Pauleto ARC, Pereira MLT, Cyrino EG. Saúde bucal: uma revisão crítica sobre programações educativas para escolares. Cien Saude Colet 2004; 9(1):121-130.

Artigo apresentado em 21/08/2014

Aprovado em 13/12/2014

Versão final apresentada em 15/12/2014 